\title{
Quantitative Angiocardiographic Recognition of Atypical Form of Tetralogy of Fallot with Absent Pulmonic Valve
}

\author{
Ali Yazdanyar, M.D., Mohammad H. Safavian, M.D., \\ F.A.C.S., ${ }^{*}$ Iraj Nazarian, M.D., F.G.A.P., ${ }^{* *}$ and \\ Jami G. Shakibi, M.D., F.A.C.C.
}

\section{SYMMARY}

A 9-year-old boy is reported who had tetralogy of Fallot, rudimentary pulmonic valve, and supravalvar pulmonic stenosis. The patient lacked almost all of the clinical findings commonly encountered in this syndrome. Thus he never had signs of a large left-to-right shunt, or congestive heart failure. He had no murmur of pulmonic regurgitation and his pulmonary artery was not large on chest roentgenogram. Angiocardiography revealed moderate main pulmonary arterial dilation. The lack of the usual manifestations of tetralogy of Fallot with absent pulmonic valve was due to supravalvar pulmonic stenosis, acting as a natural pulmonary artery band.

A quantitative angiocardiographic study was undertaken in order to find a clue for the diagnosis of these atypical cases. The ratio of the transverse diameters of the main pulmonary artery and the aortic root as measured on lateral angiocardiograms in 31 children with uncomplicated tetralogy of Fallot was $0.70 \pm 0.22$, whereas this ratio was 1.70 in this particular patient $(p<0.001)$. It is concluded that a quantitative evaluation of the ratio of the transverse diameter of the main pulmonary artery to the aortic root on lateral angiocardiogram allows differentiation of tetralogy of Fallot with absent pulmonic valve associated with supravalvar pulmonic stenosis from uncomplicated forms of tetralogy of Fallot.

\section{Additional Indexing Words:}

Pulmonary artery size Pulmonary artery anomalies

From the Departments of Pediatric Cardiology, Cardiovascular Surgery,* and Pathology, ${ }^{* *}$ Queen Pahlavi Cardiovascular Medical Center, Teheran, Iran.

This work was partly supported by the general research fund of the Queen Pahlavi Cardiovascular Medical Center.

Reprint requests to: J.G. Shakibi, M.D., Queen Pahlavi Cardiovascular Medical Genter, Shemiran, P.O. Box 33-423, Teheran, Iran.

Received for publication May 3, 1977. 


\begin{abstract}
A
BSENT or rudimentary pulmonic valve is a rare congenital cardiac defect that may occur as an isolated lesion or more commonly in combination with other anomalies of the heart. The most frequently associated lesion is tetralogy of Fallot (TF). ${ }^{1)}$ The usual clinical picture of TF with absent pulmonic valve is remarkable by gross pulmonary regurgitation, aneurysmal pulmonary arteries and history of congestive heart failure.

The purpose of this paper is to present a patient with $\mathrm{TF}$, rudimentary pulmonic valve and supravalvar pulmonic stenosis, who lacked most of the usual clinical findings commonly observed in this syndrome. A quantitative angiocardiographic clue is presented for facilitating the diagnosis of the atypical forms of this syndrome.
\end{abstract}

\title{
Materials And Methods
}

A. Case history: G.F. 778, a 9-year-old boy was admitted to the Queen Pahlavi Cardiovascular Genter, for cardiac evaluation. He was cyanotic since early infancy. He complained of shortness of breath and dyspnea on exertion. He squatted frequently, but had no history of cyanotic spells. He was the result of a

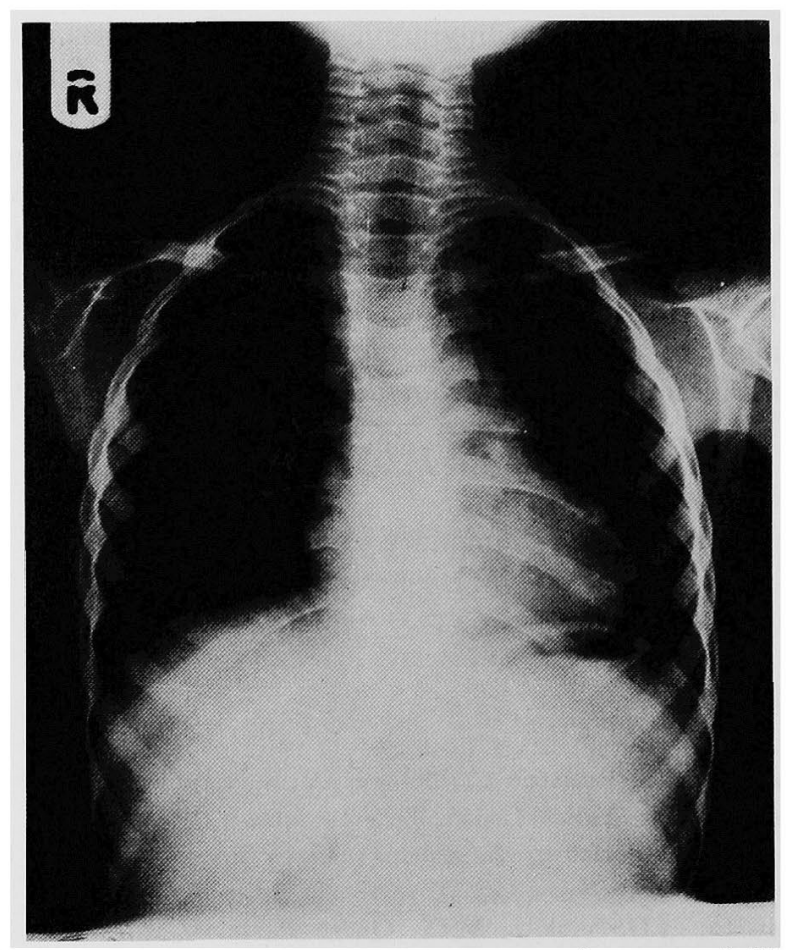

Fig. 1. Chest roentgenogram. Note absence of aneurysmal dilation of the pulmonary artery and its main branches. 
normal pregnancy and delivery. He was never in heart failure. His family history was noncontributory. Physical examination revealed a small cyanotic boy with pulse rate of $100 / \mathrm{min}$, respiratory rate of $25 / \mathrm{min}$, and blood pressure of $100 / 70 \mathrm{mmHg}$. His height was $11 \mathrm{~lm}$ and his weight was $14 \mathrm{Kg}$. Examination of the heart revealed a systolic thrill at left lower sternal border. Right ventricular heave was palpable. On auscultation S1 was normal, but P2 was absent. There was no S3, nor systolic ejection click. A grade 4/6 systolic ejection murmur was present at the third left intercostal space. There was no diastolic murmur. The abdomen was unremarkable. Peripheral pulses were normal. Clubbing of finger- and toenails was obvious. Chest roentgenogram revealed a normal cardiothoracic ratio. Right ventricle and right atrium were enlarged. Pulmonary vascular markings were reduced on both sides. Aortic arch was left-sided (Fig. 1). Electrocardiogram revealed normal sinus rhythm with a rate of $100 / \mathrm{min}$. QRS axis was at 100 degrees. Right atrial and right ventricular hypertrophy were present. Vectorcardiogram showed right ventricular hypertrophy with clockwise horizontal plane QRS-loop. Cardiac catheterization revealed systemic venous and arterial oxygen desaturation. The arterial $\mathrm{PO}_{2}$ was $40.2 \mathrm{mmHg}$ and saturation was $70 \%$. Pulmonary venous saturation was $90 \%$. Right ventricular pressure was $100 / 0-6 \mathrm{mmHg}$. Simultaneous aortic pressure was $100 / 72$ (mean 85 ) $\mathrm{mmHg}$. Pulmonary artery could not be negotiated. Angiocardiogram of the right ventricle in anteroposterior and lateral positions revealed a heavily trabeculated right ventricle with severe infundibular pulmonic stenosis. The aorta was opacified via a ventricular septal defect. The aortic root was about $70 \%$ dextroposed. The left ventricle was of adequate size. The main pulmonary artery was dilated, however the right and left branches were small. The dilatation of the main pulmonary artery was thought to represent poststenotic dilatation. The patient underwent total correction. The ventricular septal defect was closed with a patch. Right ventricular outflow tract was resected. A membrane was noted at the site of the pulmonary artery bifurcation narrowing the ostia of both right and left pulmonary arteries. The membrane was resected and the incision was closed. The systemic pressure was low and right ventricular and main pulmonary arterial pressures were suprasystemic. The patient was again put on cardiopulmonary bypass and the remnant of the occluding membrane was resected. A number 12 Hegar dilator was easily passed through both pulmonary arteries. A number 19 Björk-Shiley valve was placed at the pulmonic valve ring and a pericardial patch was sewn in the right ventricular outflow tract thus accommodating the valve strut. Following this procedure the right ventricular pressure and the main pulmonary artery systolic pressures were $70 \mathrm{mmHg}$ and there was $34 \mathrm{mmHg}$ gradient between the main pulmonary artery and both right and left main branches. The systemic pressure was low and the patient came off bypass with considerable difficulty. Shortly after the operation the patient developed profuse generalized bleeding. The bleeding could not be controlled and the patient expired several hours later. At postmortem examination the ostium of the right and left pulmonary arteries were patent. The occluding membrane was completely resected. The main pulmonary artery was aneurysmal, however the right and left pulmonary artery branches were small. The prosthetic valve was well-seated. Just above the prosthesis there were 2 pulmonary valve leaflets. One was well developed, the other was rudimentary and the third cusp was missing. The outflow and ventricular septal patches were intact. There 


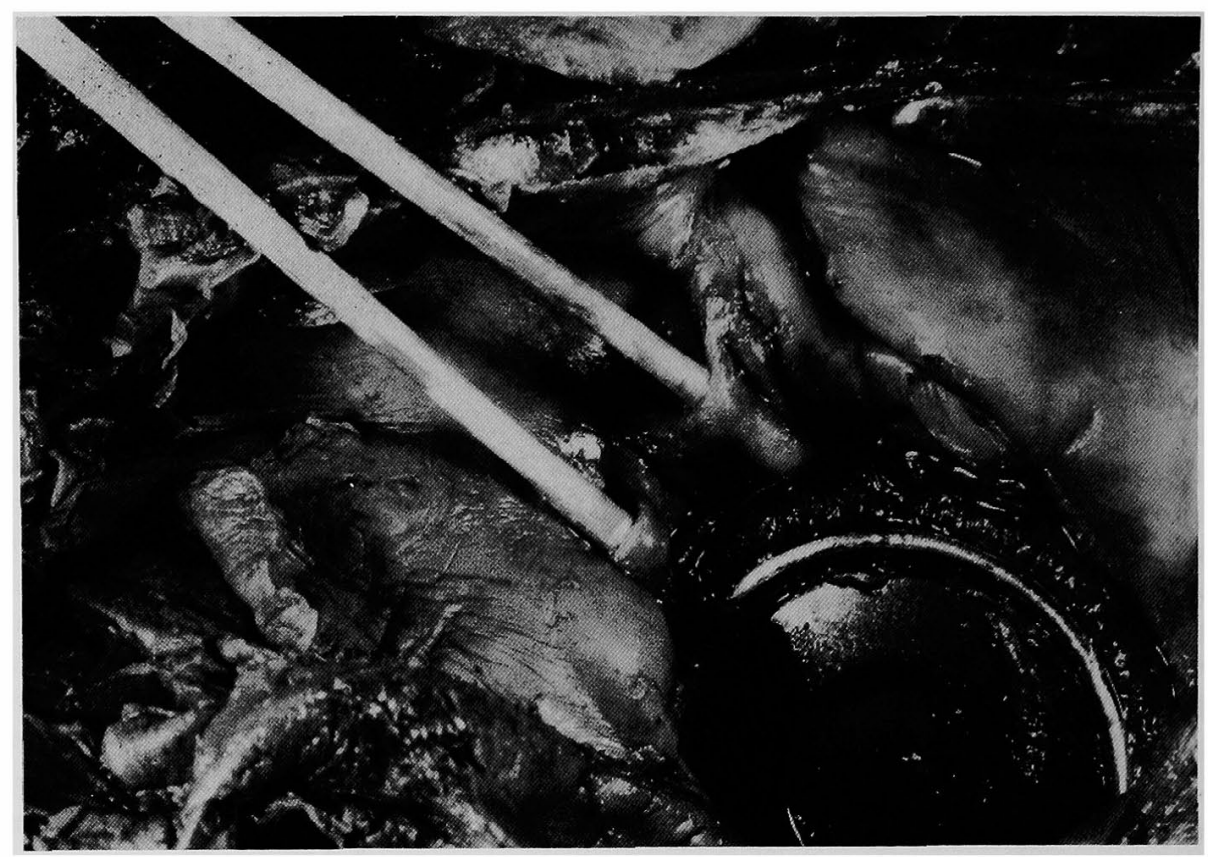

Fig. 2. Pathological specimen. Note the prosthesis and rudimentary pulmonary valve cusps (probes). The main pulmonary artery is dilated. The ostium of the left pulmonary artery is marked by an arrow.

was evidence of generalized visceral bleeding suggestive of disseminated intravascular coagulation (Fig. 2).

B. Control material: To determine the variability of the size of the main pulmonary artery in $\mathrm{TF}$, we reviewed the biplane angiocardiograms of 65 children aged between 4 months to 15 years. Thirty-one biplane angiocardiograms allowing accurate quantitative evaluation of the great arteries were chosen. The diameters of the main pulmonary artery and aorta were measured in the lateral projection. The maximum transverse diameter of the main pulmonary artery and the diameter of the aorta just above the sinus of Valsalva were measured and the result was expressed as pulmonary artery/aortic (PA/Ao) ratio. The PA/Ao ratio of the patient with $\mathrm{TF}$, rudimentary pulmonic valve and supravalvar pulmonic stenosis was compared with the control group, using routine statistical methods.

\section{Results}

The ratio of the maximum transverse diameter of the main pulmonary artery to the transverse diameter of the aortic root in 31 children with $\mathrm{TF}$ was $0.70 \pm 0.02$ (SD) (SE of the mean=0.04). The $99 \%$ confidence limit was $0.70 \pm 0.1032$. The highest ratio observed in this group of children was a single case with a ratio of 1.04 . The PA/Ao ratio in the case with $\mathrm{TF}$, rudi- 
mentary pulmonic valve and supravalvar pulmonic stenosis was 1.70 . This value is significantly different from the ratio observed in the uncomplicated forms of $\mathrm{TF}(\mathrm{p}<0.001)$.

\section{Discaussion}

Absent or rudimentary pulmonic valve is a rare anomaly which is commonly associated with other congenital cardiac defects. It has been stated that it is present in 3-6\% of autopsy cases of TF.1,2) Other associated lesions are ventricular septal defect, patent ductus arteriosus, aneurysm of the aortic sinus of Valsalva, complete atrioventricular canal, tricuspid atresia, mitral atresia, and double outlet right ventricle. ${ }^{3)}$ It occurs more commonly in males with a male/female ratio of almost $2 / 1$.

The usual manifestations of absent pulmonic valve make it an easily recognizable entity. However, complicated cases such as the patient presented in this report can only be diagnosed by a critical quantitative study of the angiocardiogram. The classical form of absent pulmonic valve presents with signs of congestive heart failure during infancy, due to a large left-toright shunt. These patients are usually very sick and if untreated die before 18 months of age. ${ }^{4-6)}$ On auscultation a to-and-fro murmur is present, the diastolic murmur being due to pulmonic regurgitation. The second heart sound is single, due to inaudible pulmonary component. The chest roentgenogram is remarkable by cardiomegaly and greatly dilated main pulmonary artery and its main right and left branches. As some of these patients grow older, they improve and develop a balanced bidirectional shunt or a predominant right-to-left shunt. Seventeen of the 18 patients with TF and absent pulmonic valve reported by Stafford et al were of this type and had predominant left-to-right shunt during infancy."

Our patient was remarkable for the lack of all of the signs enumerated above, except for moderate main pulmonary arterial dilatation and absence of P2. Thus our patient never had signs or symptoms suggestive of a left-toright shunt or congestive heart failure, even during infancy. He had no diastolic murmur of pulmonic regurgitation. His heart was not large and on angiocardiography only main pulmonary artery was moderately dilated. The reasons and explanations for these atypical findings are: 1) Severe infundibular stenosis and 2) Supravalvar pulmonic stenosis. Severe infundibular stenosis tends to reduce pulmonic regurgitation, ${ }^{3)}$ thus absence of a diastolic murmur can be accounted for. Presence of supravalvar stenosis prevented large left-to-right shunt and therefore the occurrence of congestive heart failure and cardiomegaly. Also because of limiting of the magnitude of the flow 
it prevented aneurysmal dilatation of the right and left pulmonary artery main branches. Thus supravalvar pulmonic stenosis in this case had acted as a natural pulmonary artery band. As Venables suggested, pulmonary artery banding as a palliative procedure in this entity would not only reduce the left-to-right shunt, but also limit the regurgitant flow. ${ }^{8)}$

Therefore in order to make a correct diagnosis of this complicated form of absent pulmonary valve and supravalvar pulmonic stenosis, a clue was looked for. As described under the methods a critical quantitative study of the angiocardiograms in children with uncomplicated $T F$ revealed that the ratio of the maximum transverse diameter of the pulmonary artery and aortic root in lateral angiocardiogram was $0.70 \pm 0.22$ (99\% confidence limits $0.5968-$ $0.8032)$, whereas this ratio was equal to 1.70 in this particular patient $(\mathrm{p}<$ 0.001 ). In a report by Aziz et al on the $\mathrm{PA} / \mathrm{Ao}$ ratio in children and infants with transposition of the great arteries, it was found that this ratio equaled $0.97 \pm 0.18$ in those with intact ventricular septum, whereas in the cases with associated ventricular septal defect or patent ductus arteriosus it was $1.13 \pm$ 0.19.9) Thus even in congenital heart defects leading to increased pulmonary blood flow this ratio did not exceed 1.32. Therefore one should be extremely suspicious and consider rudimentary or absent pulmonic valve in any patient presenting with the clinical and/or angiocardiographic features of TF, but with a PA/Ao ratio exceeding 0.80 . The only group of patients which should be differentiated from the above is those with ventricular septal defect and pulmonic valvar stenosis, and poststenotic main pulmonary artery dilatation. In these patients, the anatomy and peculiar orientation of the right ventricular outflow tract, visualization of the pulmonary valves and lack of right and left pulmonary artery dilatation are sufficient to allow distinction between these two entities.

\section{REFERENCES}

1. Lev M, Eckner FAO: Pathological anatomy of tetralogy of Fallot and its variations. Dis Chest 45:251, 1964

2. Nagao GI, Daoud GI, McAdams AJ et al: Cardiovascular anomalies associated with tetralogy of Fallot. Am J Cardiol 20: 206, 1967

3. D'Gruz IA, Lendrum BL, Novak G: Congenital absence of the pulmonary valve. Am Heart J 68 : 728, 1964

4. Osman MZ, Meng CL, Girdany BR: Congenital absence of the pulmonary valve: Report of eight cases with review of the literature. Am J Roentgen 106: 58, 1969

5. Durnin RE, Willner $R$, Virmanis $S$ et al: Pulmonary regurgitation with ventricular septal defect and pulmonic stenosis-tetralogy of Fallot variant. Am J Roentgen $106: 42,1969$

6. Waldhausen JA, Friedman $\mathrm{S}$, Nicodemus $\mathrm{H}$ et al: Absence of the pulmonary valve in patients with tetralogy of Fallot: Surgical management. J Thorac Cardiovase Surg 57: 669, 1969 
7. Stafford EG, Mair DD, McGoon DC et al: Tetralogy of Fallot with absent pulmonary valve. Surgical considerations and results. Circulation 47, 48 (suppl III): III-24, 1973

8. Venables AW: Absence of the pulmonary valve with ventricular septal defect. Brit Heart J 24: 293, 1962

9. Aziz KU, Nanton MA, Kidd L et al: Variations in the size and distensibility of the pulmonary arteries in d-transposition of the great arteries. Am J Cardiol 38: 452, 1976 